\title{
A method for detecting self-position using 3 modulated LEDs and photodiode
}

\author{
Takumi Matoba $^{\text {a, }}$, Seiichi Serikawa ${ }^{\text {a }}$ \\ ${ }^{a}$ Department of Electrical and Electronic Engineering, Kyushu Institute of Technology, Kitakyushu, Japan \\ *Corresponding Author: matoba.takumi683@mail.kyutech.jp
}

\begin{abstract}
In recent years, the decrease in working population due to declining birthrate and aging population has become a problem in Japan. A line trace type automatic guided vehicle is used as a solution to the problem. However, the method has some disadvantages that the route cannot be changed easily and that it cannot move while avoiding obstacles on the route. Therefore, we focused on drones that can move freely in space. As a method of autonomous movement of a drone in a non-GPS environment such as in a warehouse, a method using a stereo camera is mainly used. However, there are disadvantages in terms of cost and accuracy. Thus, in order to improve these disadvantages, we focused on a method for detectiong self-position using changes in the illuminance of light. We propose a method for detectiong three-dimensional self-position that uses three LEDs and photodiodes which are modulated to different frequencies. We experimented to check the validity of the method, and showed that this is an effective method for detecting the selfposition of the drone in a room. It has the advantages that it can move on any route just by installing three LEDs, and that the cost is low.
\end{abstract}

Keywords: photodiode, method, drone, self-position

\section{Introduction}

In recent years, working population has been declining in Japan due to declining birthrate and aging population [1]. Therefore, automatic guided vehicles are used to solve the problem. Currently, there is a line trace type as an automatic guided vehicle that is mainly used. In this method, when changing the route, it is necessary to remove a line marker that serves as a mark and attach it again. Also, if there is an obstacle on movement path, it cannot be avoided. Therefore, we focused on drones. Since a drone can move freely in space, movement route can be easily changed. In addition, obstacles on route can be easily avoided. Therefore, it can be expected as a solution to disadvantages of conventional line trace type robot. Currently, drone-type autonomous mobile robots mainly use GPS. However, since it is difficult to receive GPS radio waves in a room such as a warehouse or in a tunnel, the error may become large or measurement may not be possible. Therefore, stereo cameras are mainly used in non-GPS environments such as indoors. However, stereo cameras have some disadvantages that they cannot be easily introduced due to the complicated system and that they are expensive. Therefore, we focused on the self-position detection method using the change in light illuminance as a new autonomous movement method in a new non-GPS environment that can improve these disadvantages.

In this study, we propose a new method for detecting three-dimensional self-position using three different frequency-modulated LEDs, photodiodes, and ultrasonic sensors.

\section{Principle}

This chapter describes the principles used in this method.

\section{1 illuminance measurement in front direction}

An illuminance in front direction is defined as $E_{\max }$. The Illuminance changes with height. The illuminance $E_{\max i}$ in the front direction is measured while changing height $h_{i}$. In addition, an illuminance decreases as a direction leaves from front direction. This light distribution follows the cos4 law. From the above, in order to detect the self-position in three dimensions, it is necessary to measure frontal illuminance $E_{\text {maxi }}$ of any high $h_{i}$ in advance. The relationship between illuminance and distance is shown in Figure 2.1 and (2.1). At this time, the distance from the LED is defined as $h_{n}$, and the 
illuminance is defined as $E_{\operatorname{maxn}}$.

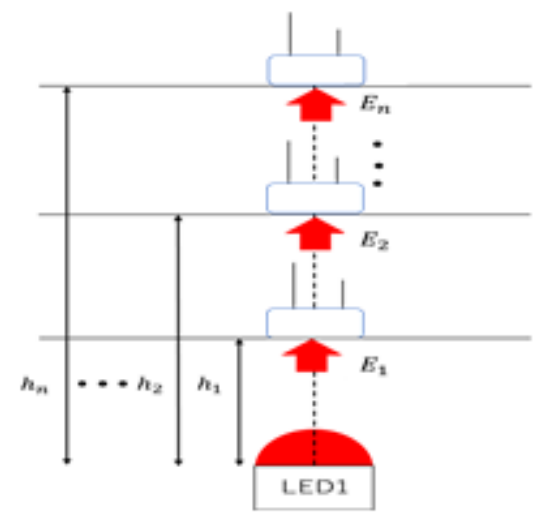

Fig. 2.1 Relationship between illuminance and distance in the central direction.

$$
E_{\operatorname{maxn}}=\frac{h_{n-1}^{2}}{h_{n}^{2}} E_{\max n-1}
$$

From Fig 2.1 and Eq.(2.1), it can be seen that illuminance decreases as height increases. In addition, it is necessary to measure the illuminance with each LED.

Next, as shown in Fig. 2.2, three LEDs (LED1, LED2, LED3) are set on floor, and the coordinates $(a, b),(c, d),(e$, $f)$ and the three LEDs are modulated. The frequencies $f_{1}, f_{2}$, and $f_{3}$ of three LEDs are given to a drone in advance.

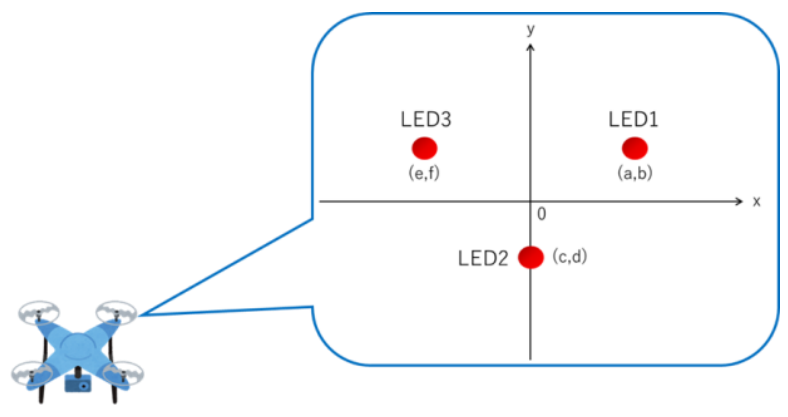

Fig. 2.2 Prior information to give to a drone.

\subsection{Self-position detection in $2 \mathrm{D}$ space}

Self-position detection in two-dimensional space is possible by using cos4 law of illuminance. By using the light receiving illuminance of one LED as shown in Fig. 2.3, it is possible to identify that a drone is on circumference of radius. However, self-position of drone cannot be specified. Also, using two LEDs as shown in Figure 2.4, the drone is the intersection of two circles, and it can be estimated that it is located at one of the two points. However, since there are two candidates, it is not enough. Therefore, it is necessary to use illuminance received from three LEDs. As shown in Fig. 2.5, the self-position of the drone in two-dimensional space is obtained by finding the coordinates of intersections of three circles.

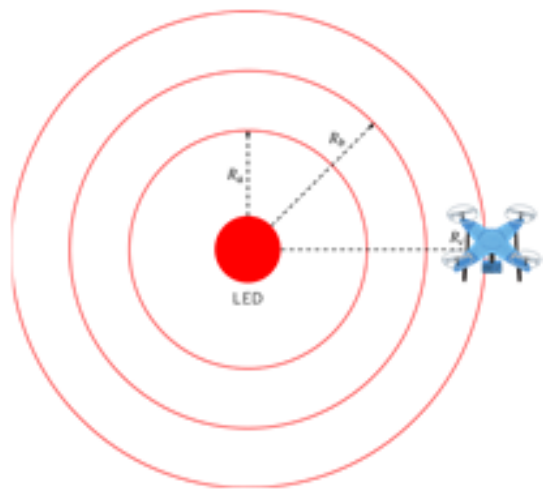

Fig. 2.3 Distance obtained by one LED.

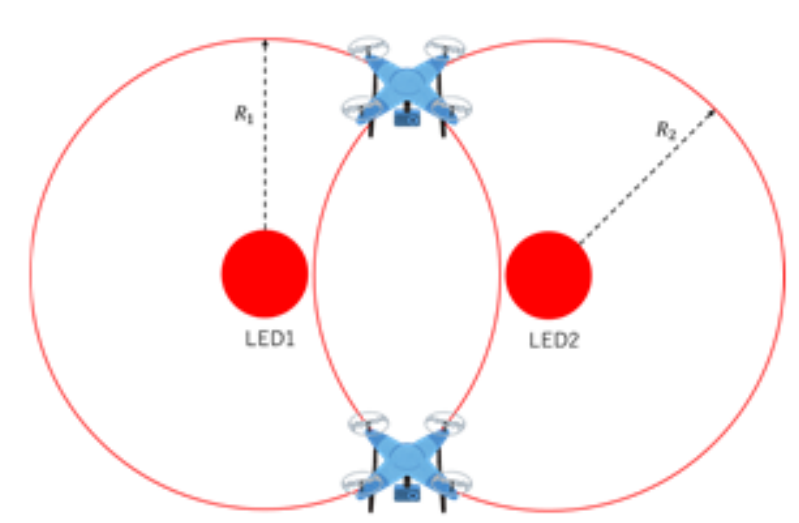

Fig. 2.4 Two candidates for self position obtained by two LEDs.

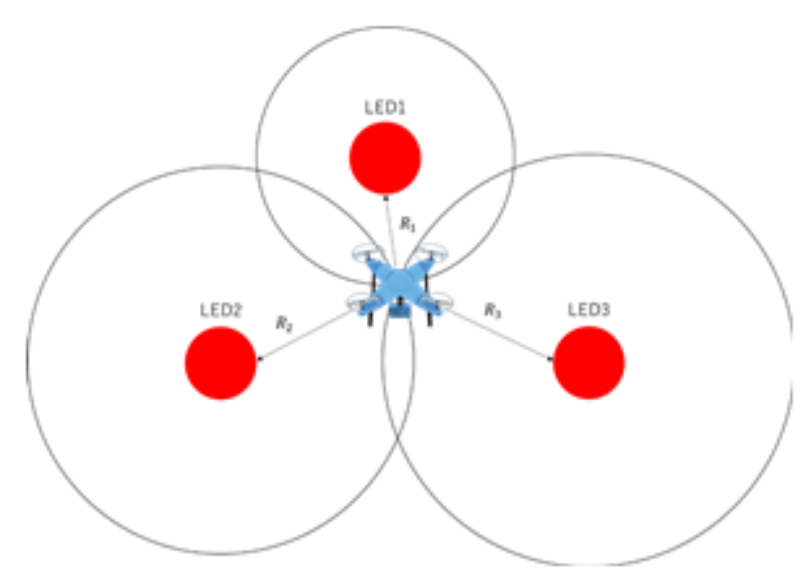

Fig. 2.5 Self position obtained by three LEDs.

Next, this can be expressed by an equation as follows. Let $E_{\text {max }}$ be the illuminance in the front direction measured in Chapter 2.1, and $E_{i}$ be the illuminance measured at a point leaved from front direction. Here, the angle $\theta$ is defined as the angle between the measurement point and front direction. From the cos4 law of illuminance, the following two equations hold. 


$$
\begin{array}{r}
E_{i}=E_{\max } \cos ^{4} \theta \quad(i=1,2,3) \\
\theta=\cos ^{-1}\left(\sqrt[4]{\frac{E_{i}}{E_{\max }}}\right) \quad(\mathrm{i}=1,2,3)
\end{array}
$$

If $R_{i}$ is defined as the distance from each LED to the measurement point, and $\mathrm{Z}$ is defined as the height of the measurement point, the following equation holds.

$$
R_{i}=\mathrm{Z} \tan \theta(i=1,2,3)
$$

From Eqs. (2.3) and (2.4), the following equation holds.

$$
\begin{aligned}
& R_{1}=\mathrm{Z} \tan \cos ^{-1}\left(\sqrt[4]{\frac{E_{1}}{E_{\max }}}\right) \\
& R_{2}=\mathrm{Z} \tan \cos ^{-1}\left(\sqrt[4]{\frac{E_{2}}{E_{\max }}}\right) \\
& R_{3}=\mathrm{Z} \tan \cos ^{-1}\left(\sqrt[4]{\frac{E_{3}}{E_{\max }}}\right)
\end{aligned}
$$

If the coordinates of each LED are defined as $(a, b),(c, d)$, and $(e, f)$, the following equation holds.

$$
\begin{gathered}
R_{1}{ }^{2}=(x-a)^{2}+(y-b)^{2} \\
{R_{2}}^{2}=(x-c)^{2}+(y-d)^{2} \\
R_{3}{ }^{2}=(x-e)^{2}+(y-f)^{2} \\
R_{1}{ }^{2}=x^{2}-2 a x+a^{2}+y^{2}-2 b y+b^{2} \\
{R_{2}}^{2}=x^{2}-2 c x+c^{2}+y^{2}-2 d y+d^{2} \\
{R_{3}}^{2}=x^{2}-2 e x+e^{2}+y^{2}-2 f y+f^{2}
\end{gathered}
$$

From these equations, the self-position $(\mathrm{x}, \mathrm{y})$ of the drone is as follows.

$$
\begin{aligned}
& x=\frac{\left\{\left(R_{1}{ }^{2}-R_{2}{ }^{2}\right)-\left(a^{2}+b^{2}-c^{2}-d^{2}\right)\right\}(d-f)+\left\{\left(R_{3}{ }^{2}-R_{2}{ }^{2}\right)+\left(c^{2}+d^{2}-e^{2}-f^{2}\right)\right\}(b-d)}{2\{(-a+c)(d-f)+(b-d)(c-e)\}} \\
& y=\frac{\left\{\left(R_{1}{ }^{2}-R_{2}{ }^{2}\right)-\left(a^{2}+b^{2}-c^{2}-d^{2}\right)\right\}(c-e)+\left\{\left(R_{3}{ }^{2}-R_{2}{ }^{2}\right)+\left(c^{2}+d^{2}-e^{2}-f^{2}\right)\right\}(a-c)}{2\{(-b+d)(c-e)+(a-c)(d-f)\}}(2 .)
\end{aligned}
$$

By substituting Eqs (2.8) and (2.9) into Eqs. (2.10), (2.11), and (2.12), the two-dimensional coordinates (x,y) at the height of $z$ can be obtained. Thus, by continuously measuring coordinates using drone, it is possible to detect self-position at 2D space.

\subsection{Self-position detection in 3D space}

In order to detect the self-position in three dimensions, it is necessary to measure height $z$ from floor and the illuminance $E_{\max }$ in front direction. The reason is that $E_{\text {max }}$ depends on $z$. Therefore, by letting drone know the ralationship between height $z$ and $E_{\max }$ in advance, it is possible to detect the two-dimensional self-position $(x, y)$ at the height $z$. Then, by combining the two-dimensional selfposition $(x, y)$ and the height $z$ of this drone, it is possible to detect the three-dimensional self-position $(x, y, z)$. This is the principle that enables self-position detection in three dimensions.[2]

\section{Proposed Method}

\subsection{System configuration}

In this system, three different frequency-modulated LEDs are set on the floor. Then, the light from the LED is received by the photodiode attached to the drone. The voltage proportional to received illuminance is input to the Raspberry Pi via an amplifier circuit and A / D converter. In addition, the height $\mathrm{z}$ of the drone is input to the Raspberry $\mathrm{Pi}$ using an ultrasonic sensor. In this experiment, the height $\mathrm{z}$ is regarded that the height is measured by the ultrasonic sensor. Self-position detection in three dimensions is practiced by calculating with Raspberry Pi using three lightreceiving illuminances and heights. In this system, it consists of a light emitting part and a light receiving part. The LEDs and Arduino used for frequency modulation are called the

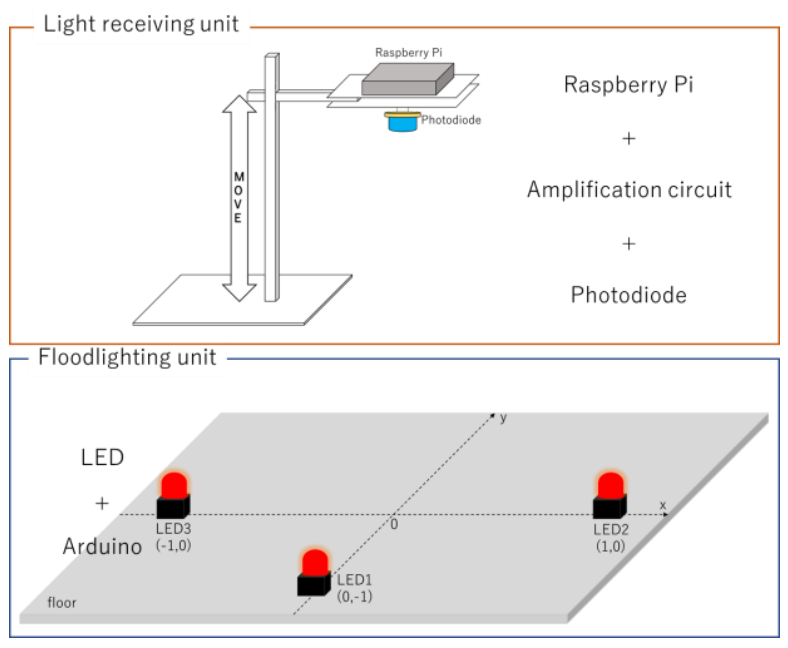

Fig. 3.1 System configuration.

light emitting part. The photodiode, amplifier circuit, A / D converter, and Raspberry Pi are called the light receiving part. The configuration is shown in Figure 3.1.

\subsection{Light emitting part}

Light emitting part consists of a frequency modulation circuit and red LEDs. The circuit configuration is shown in Fig. 3.2. Since the frequency modulation circuit consists of Arduino and transistors, it is possible to generate arbitrary 
frequency by changing the programming of Arduino.

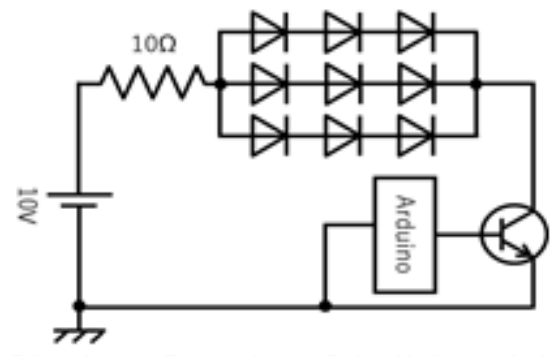

Fig. 3.2 Circuit configuration of the light emitting part.

In this study, LED1, LED2, and LED3 are made as three light emitting parts, and each is modulated at three different frequencies. The frequency $f_{1}$ of LED1 was $2.0 \mathrm{kHz}$, the frequency $f_{2}$ of LED2 was $2.5 \mathrm{kHz}$, and the frequency $f_{3}$ of LED3 was $3.0 \mathrm{KHz}$.

\subsection{Light receiving part}

Figure 3.3 shows the circuit configuration of the light receiving circuit. It consists of a photodiode, a signal amplifier circuit and an A / D converter. The light receiving part consists of the light receiving circuit and Raspberry Pi.

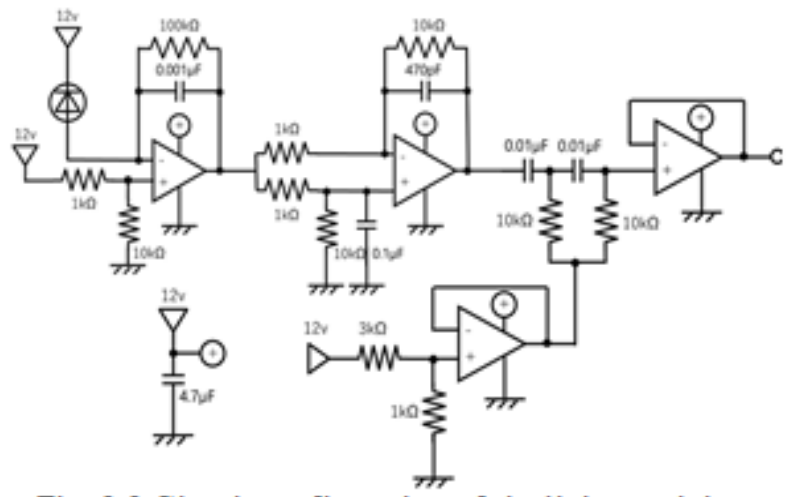

Fig. 3.3 Circuit configuration of the light receiving part.

The operation of light receiving circuit will be explained. At first, LED light is received by photodiode. Then, the received illuminance is converted into an electric current, amplified by an amplifier circuit, and converted into a digital signal by an A / D converter.

Next, Raspberry $\mathrm{Pi}$ gets the information of three illuminances and height $\mathrm{z}$ from floor, and estimates threedimensional self-position. The flowchart is shown in Fig. 3.4.
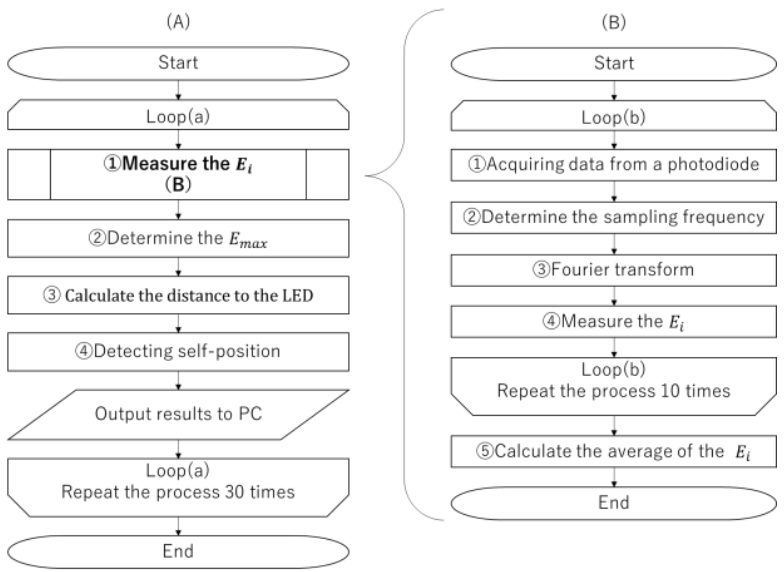

Fig. 3.4 Algorithm for 3D self-position estimation.

Algorithm (A) is described as follows.

(1) Extract the frequency components of $2.0 \mathrm{kHz}, 2.5 \mathrm{kHz}$, and $3.0 \mathrm{kHz}$, and gets the maximum amplitude of each frequency component. The Details are explained in algorithm (B).

(2) Calculate the illuminance $E_{\max }$ in the upper direction from the floor. Equation (2.1) is used for the calculation. In this experiment, only heights of $1.0 \mathrm{~m}$ and $1.5 \mathrm{~m}$ were tested.

(3) Substitute the amplitude values $E_{i}$ and illuminance $E_{\text {max }}$ obtained in (1) and (2) into Eqs. (2.5)-(2.7) to calculate the distances $R_{1}, R_{2}$, and $R_{3}$.

(4) By substituting the distance value calculated in (3) into Eqs. (2.11) and (2.12), the two-dimensional self-position coordinates $(x, y)$ are calculated. Then, from the height $z$ gotten in (2), the three-dimensional coordinates $(x, y, z)$ is obtained.

Next, algorithm (B) is explained.

(1) Acquire $4096 \mathrm{~A} / \mathrm{D}$ converted illuminance data.

(2) Calculate the sampling time. Let the sampling time be $d t$ and the illuminance data acquisition time be $t, d t$ is given by the following equation.

$$
\mathrm{dt}=\frac{t}{4096}
$$

(3) Practice discrete Fourier transform on the illuminance data of (1) using the sampling frequency of (2).

(4) Extract the amplitude values $E_{i 1}, E_{i 2}$, and $E_{i 3}$ at $2.0 \mathrm{kHz}, 2.5 \mathrm{kHz}$, and $3.0 \mathrm{kHz}$ from the values in (3).

(5) Repeat (1)-(4) 10 times. Let the average value be the amplitude value at each frequency.

\section{Experiment}

4.1 Experiment 1: Measurement of illuminance in the 


\section{front direction}

In this system, the illuminance in the front direction of the LED was measured.

\subsubsection{Experimental method}

As for the LED, a total of 9 red LEDs, 3x3, were connected and used as one LED. Figure 4.1 shows the positional relationship between the light emitting part and the light receiving part. The illuminance $E_{\max }$ was measured by changing the distance between the light emitting part and the light receiving part from $0.5 \mathrm{~m}$ to 2.5 $\mathrm{m}$.

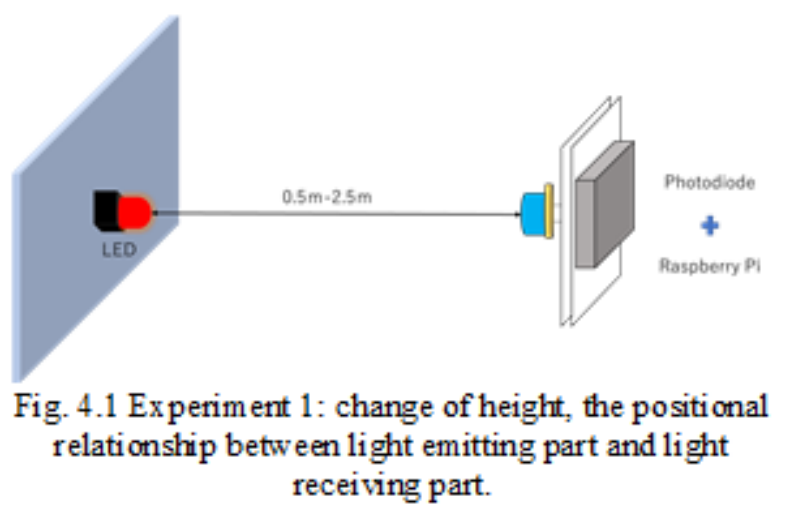

\subsubsection{Result}

Table 4.1 shows the measurement results of the illuminance $E_{\max }$ at the distances of the three LEDs at each frequency.

Table 4.1 Result of Experiment 1.

\begin{tabular}{|c|c|c|c|c|}
\hline & \multicolumn{3}{|c|}{ Fumin snoe(V) } \\
\hline & & LEDI & LEDZ & LEDS \\
\hline \multirow{5}{*}{$\begin{array}{c}\text { Distance } \\
\text { (m) }\end{array}$} & 0.5 & 0234 & 0.25 & $0.2 n$ \\
\hline & 1.0 & 0.071 & 0.055 & 0.065 \\
\hline & 1.5 & 0.033 & 0.009 & 0.038 \\
\hline & 20 & 0.020 & 0.025 & 0.023 \\
\hline & 2.5 & 0.015 & 0.017 & 0.025 \\
\hline
\end{tabular}

This value is used as the illuminance in the front direction in Experiment 2.

\subsection{Experiment 2: Self-position detection in 3D space}

To confirm the effectiveness of this system, we verified the accuracy of 3D self-position detection.

\subsubsection{Experimental method}

Figure 4.2 shows the positional relationship between the light emitting part and the light receiving part.
As shown in Fig. 4.2 (a), three LEDs are installed on the floor. The coordinates of each LED are $(x, y)=(0 m,-1 \mathrm{~m})$ for LED1, $(\mathrm{x}, \mathrm{y})=(1 \mathrm{~m}, 0 \mathrm{~m})$ for LED2, and $(\mathrm{x}, \mathrm{y})=(-1 \mathrm{~m}, 0 \mathrm{~m})$ for LED3. From this page onward, the description of unit of [m] is omitted. LED1 is frequency-modulated to $2.0 \mathrm{kHz}$, LED2 to $2.5 \mathrm{kHz}$, and LED3 to $3.0 \mathrm{kHz}$.

The measurement points are shown in Fig. 4.1 (b). As can be seen from this figure, there are 25 measurement points. At first, the height $\mathrm{z}$ is set to 1.0 . The coordinates of 25 points are detected by moving 0.5 in the range of -1 to 1 in the $x$ direction and 0.5 in the range of -1 to 1 in the $y$ direction. Next, the height $\mathrm{z}$ is set to 1.5 . Similarly, by moving 0.5 in each direction, the coordinates of 25 points are detected. Each coordinate is detected 30 times, and the average is regarded as the coordinate.

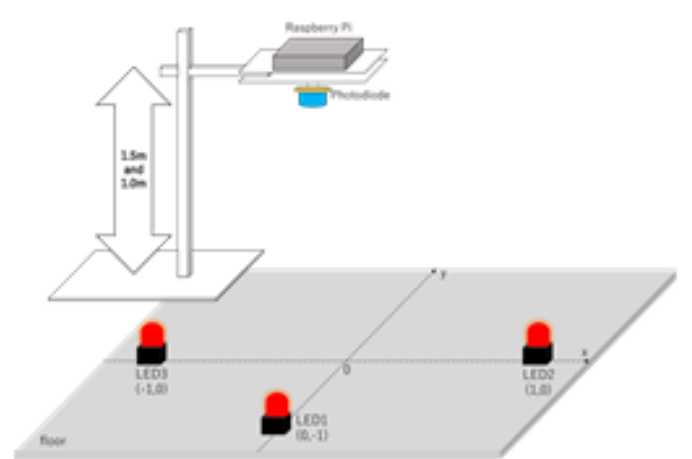

(a) Positional relationship of parts.

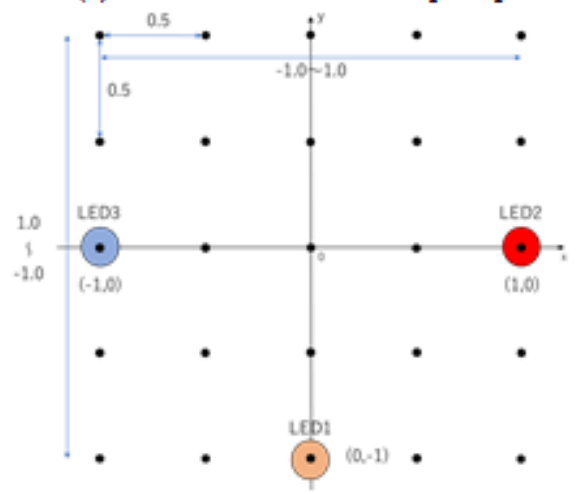

(b) Measurement point (top view)

Fig. 4.2 Experiment 2: movement to 3 directions, the positional relationship between light emitting part and light receiving part.

\subsubsection{Result}

Figure 4.3 shows the theoretical value of Experiment 2, and Figure 4.4 shows the measurement results when the height is $1.0 \mathrm{~m}$. Figure 4.5 shows the results when the height is $1.5 \mathrm{~m}$. Next, Table 4.2 shows the difference between the theoretical value and the measured value when the height is 
$1.0 \mathrm{~m}$, and Table 4.3 shows the difference between them when the height is $1.5 \mathrm{~m}$. The unit of this table is [cm].

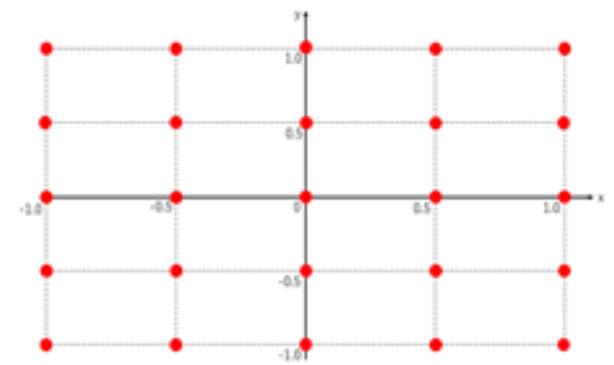

Fig. 4.3 Theoretical Coordinates of Ex periment 2.

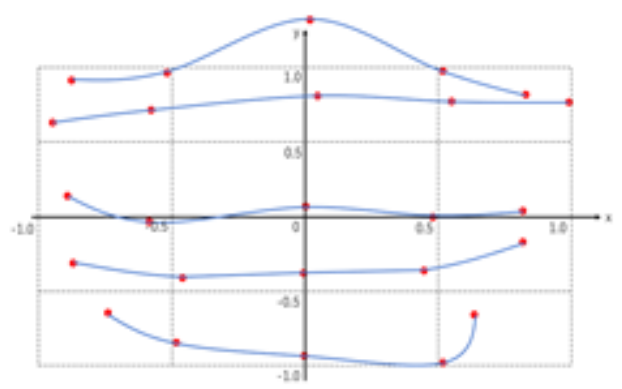

Fig. 4.4 Coordinates measured in Ex periment 2, at height $1 \mathrm{~m}$

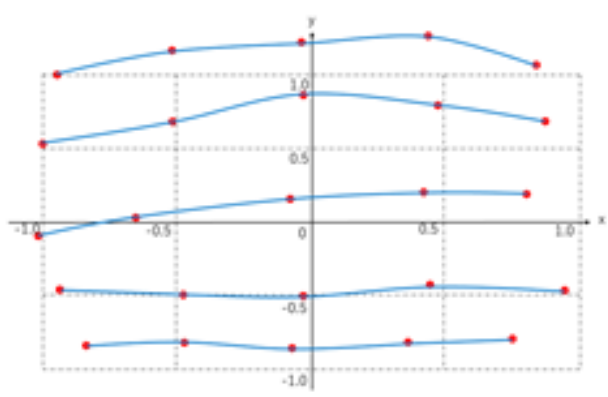

Fig. 4.5 Coordinates measured in Ex periment 2, at height $1.5 \mathrm{~m}$.

Table 4.2 Difference between experimental value and theoretical value of Experiment 2, at height $1 \mathrm{~m}$.

\begin{tabular}{|c|c|c|c|c|c|c|}
\hline \multirow{2}{*}{\multicolumn{2}{|c|}{ Gspl(cm) }} & \multicolumn{5}{|c|}{$x$} \\
\hline & & -1 & $-0 s$ & 0 & os & 1 \\
\hline \multirow{5}{*}{$y$} & 1 & 24.770 & $385\rangle$ & 32283 & 2.734 & $24 . m$ \\
\hline & Q.S & 24.32 & 23058 & 31.544 & 28.060 & 27.05 \\
\hline & 0 & 17.36 & 92223 & 7.050 & 2091 & 23.72 \\
\hline & -0.5 & 23.079 & 9892 & 12334 & 1s.coss & 3.991 \\
\hline & -1 & 4.181 & 15898 & 6.700 & 2.934 & 50.158 \\
\hline
\end{tabular}

Table 4.3 Difference between experimental value and theoretical value of Experiment 2, at height $1.5 \mathrm{~m}$.

\begin{tabular}{|c|c|c|c|c|c|c|}
\hline \multirow{2}{*}{\multicolumn{2}{|c|}{ Gspl(cm) }} & \multicolumn{5}{|c|}{$x$} \\
\hline & & -1 & -0.5 & 0 & as & 1 \\
\hline \multirow{5}{*}{$y$} & 1 & 5243 & 16058 & 22.444 & 27.294 & $17.5 \alpha$ \\
\hline & 05 & 3313 & 18271 & $356 / 1$ & 29394 & 2253 \\
\hline & 0 & $9 / 10$ & $15 \sqrt{16}$ & 17,454 & 21934 & 27,328 \\
\hline & -2.5 & 7.463 & 2206 & 3075 & 9251 & $G \gamma \mathbb{Q}$ \\
\hline & -1 & 22735 & 18060 & 15947 & 23115 & 32358 \\
\hline
\end{tabular}

\subsection{3 investigation}

From Table 4.2-Table 4.3, the maximum error is $50 \mathrm{~cm}$ when the height is $1.0 \mathrm{~m}$ and $32 \mathrm{~cm}$ when the height is $1.5 \mathrm{~m}$. However, looking at Fig. 4.4 and Fig. 4.5, there is an error of $50 \mathrm{~cm}$ around only the boundary of the measurement range $( \pm 1 \mathrm{~m}, \pm 1 \mathrm{~m})$, but otherwise it is an error of up to $30 \mathrm{~cm}$. It is said that the position can be detected correctly. Therefore, it is considered that self-position detection in three dimensions is effective for this system.

The following four factors can be considered as the causes of measurement error.

(1) LED light is not irradiated uniformly in all directions In this study, the self-position is detected based on the assumption that the LED light is irradiated uniformly in all directions. However, the light changes depending on a direction, which causes an error. It is necessary to improve the arrangement of LEDs.

(2) The circuit of the light receiving part is unstable There is a noise when amplifying illuminance from photodiode and when practicing A/D conversion. Therefore, the data fluctuates. It is necessary to make a circuit with lower noise.

(3) Setting angle of the photodiode changes

The tilt of photodiode changes due to vibration in light receiving part when moving measurement position. When actually mounting it on a drone, it can be improved by detecting tilt of photodiode using a vibration absorber or an acceleration sensor, and correcting tilt of photodiode.

(4)Processing power of is heavy

Because the software in Raspberry Pi is heavy, the hardware sometime gets hot. It affects the analog value of sensor circuit. It will be improved by simplifying algorithm and adding cooling-parts for Raspberry Pi.

\section{Conclusions}

In this paper, we propose a new method to improve the problems of conventional method for detecting self-position 
of the drone indoors. The method uses three different frequency-modulated LEDs and photodiodes. The advantages of the system are that it can specify an arbitrary route just by adopting LEDs and it does not cost.

A future issue is to improve accuracy. For the purpose, we plan to uniformly irradiate light in all directions, simplify software algorithm, and absorb vibration of photodiode.

\section{References}

(1) Mizuho Research Institute,[Declining birthrate and aging population will reduce the working population by 40\%],https://www.mizuho-

ri.co.jp/publication/research/pdf/insight/p1170531.pdf(2017 )

http://www.ric.titech.ac.jp/saneken/stereo.htm

(2) Kosuke Onishi,[Position Detection Using One

Photodiode and Multiple LED Makers],(2017) 